\title{
DIRProt: a computational approach for discriminating insecticide resistant proteins from non-resistant proteins
}

\author{
Prabina Kumar Meher', Tanmaya Kumar Sahu², Anjali Banchariya ${ }^{2,3}$ and Atmakuri Ramakrishna Rao 2*
}

\begin{abstract}
Background: Insecticide resistance is a major challenge for the control program of insect pests in the fields of crop protection, human and animal health etc. Resistance to different insecticides is conferred by the proteins encoded from certain class of genes of the insects. To distinguish the insecticide resistant proteins from non-resistant proteins, no computational tool is available till date. Thus, development of such a computational tool will be helpful in predicting the insecticide resistant proteins, which can be targeted for developing appropriate insecticides.

Results: Five different sets of feature viz., amino acid composition (AAC), di-peptide composition (DPC), pseudo amino acid composition (PAAC), composition-transition-distribution (CTD) and auto-correlation function (ACF) were used to map the protein sequences into numeric feature vectors. The encoded numeric vectors were then used as input in support vector machine (SVM) for classification of insecticide resistant and non-resistant proteins. Higher accuracies were obtained under RBF kernel than that of other kernels. Further, accuracies were observed to be higher for DPC feature set as compared to others. The proposed approach achieved an overall accuracy of $>90 \%$ in discriminating resistant from non-resistant proteins. Further, the two classes of resistant proteins i.e., detoxification-based and targetbased were discriminated from non-resistant proteins with $>95 \%$ accuracy. Besides, $>95 \%$ accuracy was also observed for discrimination of proteins involved in detoxification- and target-based resistance mechanisms. The proposed approach not only outperformed Blastp, PSI-Blast and Delta-Blast algorithms, but also achieved $>92 \%$ accuracy while assessed using an independent dataset of 75 insecticide resistant proteins.
\end{abstract}

Conclusions: This paper presents the first computational approach for discriminating the insecticide resistant proteins from non-resistant proteins. Based on the proposed approach, an online prediction server DIRProt has also been developed for computational prediction of insecticide resistant proteins, which is accessible at http://cabgrid.res.in: 8080/dirprot/. The proposed approach is believed to supplement the efforts needed to develop dynamic insecticides in wet-lab by targeting the insecticide resistant proteins.

Keywords: Insecticide resistance, SVM, Di-peptide composition, Cytochrome P450, GABA

\section{Background}

Insecticides are used to control the insects affecting the agricultural crops, parasitizing livestock, as well as to eradicate the pests transmitting dangerous infectious diseases. However, frequent application of insecticides has resulted in the resurgence of pests and appearance of resistant pest species. Insecticide resistance is the heritable change in the sensitivity of a pest population

\footnotetext{
* Correspondence: rao.cshl.work@gmail.com

${ }^{2}$ Centre for Agricultural Bioinformatics, ICAR-Indian Agricultural Statistics

Research Institute, New Delhi 110012, India

Full list of author information is available at the end of the article
}

that is reflected in the repeated failure of a product (insecticides) to achieve the expected level of control when used according to the level of recommendation for that pest species [1]. Several studies have indicated the involvement of multiple genes in conferring the resistance to many insect species [2-4]. Thus, characterization of these genes is useful to understand the development of resistance and designing new strategies to minimize the development of insecticide resistance [5].

Three major mechanisms are involved in insecticide resistance [5]: (i) detoxification of insecticides through alteration in the activities of enzymes like esterase, oxidases 
or glutathione S-transferases (GSTs) that prevents the insecticide from reaching to its site of action [6-8], (ii) Insensitivity of the insecticide target proteins $[9,10]$, because of which the insecticide no longer binds to its target $[11,12]$ and (iii) reduction in insecticide uptake due to decrease in permeability of insect cuticle [13, 14]. Though there is evidence of alteration in cuticular penetration, most of the studies have focused and evaluated the target site insensitivity and detoxification of insecticides (metabolic resistance) mechanisms. Moreover, these two mechanisms have been reported to cover a wide range of resistance levels to almost all available insecticides [9].

The cytochrome P450 family of genes in insect play an important role in the detoxification of insecticides resulted in the development of resistance to insecticides $[4,15,16]$. Besides, GSTs have also been reported to be involved in the detoxification of insecticides [17, 18]. As far as target-based mechanism is concerned, there are three main targets for conventional insecticides viz., GABA ( $\gamma$-amino butyric acid)-gated chloride ion channel, voltage-gated ion channel and acetylcholinesterases [19]. The GABA receptor is the site of target for cyclodiene (dieldrin) insecticides [20], where the resistance to dieldrin $(R d l)$ is conferred by the change of a single amino acid in GABA-gated chloride ion channel encoded by $R d l$ gene [21]. Further, knockdown resistance $(K d r)$ is one of the major forms of resistance to DDT and pyrethroid insecticides [22], which is associated with mutations in the voltage-gated sodium channel [22-25]. Acetylcholinesterase (AChE) in nerve synapses is the target protein for the insecticides like organophosphorus (e.g., malathion, fenitrothion) and carbamate (e.g., propoxur, sevin) [12]. The point mutation in the insecticide-binding site of $\mathrm{AChE}$ has been identified as the cause of insensitivity to these insecticides [26].

The above mentioned works help enable to understand the molecular mechanisms involved in the insecticide resistance. Further, the analysis of bio-molecules involved in this phenomenon has confirmed the importance of single genes in target site resistance and involvement of multi-gene families like cytochrome P450 in metabolic resistance [27]. Several studies on the effects of mutational changes in target proteins on insecticide resistance aid to the knowledge on the insect proteins involved in this process. For instance, Riveron et al. [28] demonstrated that the single amino acid change (L119F) in an up regulated GST gene, GSTe2, confers high level of metabolic resistance to DDT in the malaria vector Anopheles funestus. In another study, Nwane et al. [29] identified that two mutations at position 1014 of the $\mathrm{S}_{6}$ transmembrane segment of domain II in the voltage gated sodium channel i.e., leucine to a phenylalanine (L1014F) or to a serine (L1014S) confers resistance to DDT and pyrethroid insecticides in Anopheles gambiae.
In the recent past, several studies have identified speciesspecific insecticide resistant genes through transcriptome and expression profile analysis. Hsu et al. [30] identified 90 P450, 42 GST, 31 CoE-related genes in Bactrocera doralis, representing three major enzyme families involved in insecticide metabolism and resistance. In another study, 49 P450, 31 GST and 21 CES-specific genes of Liposcelis bostrychophila were reported to be involved in insecticide resistance, through transcriptome and differential gene expression analysis [31]. Recently, Cui et al. [32] identified relevant genes in response to flubendiamide insecticide in Asian corn borer (Ostrinia furnacalis), through de novo transcriptome and expression-profile analysis.

Though the transcriptome and expression profile analysis is one way of identifying the resistance genes, it is species specific. Moreover the expression profile analysis is expensive as well as time consuming. Thus, development of a computational tool for identifying the resistant genes independent of the species and economically as well would help in augmenting the research related to the identification of insecticide resistant genes. However, no computational tool is reported till date for the discrimination of insecticide resistant proteins from the proteins that do not confer resistance. Keeping this in view, we propose a computational approach to discriminate the insecticide resistant proteins from non-resistant proteins. The developed computational approach can be used for identification of the resistant proteins across species as well as with minimum resource (cost and time). We have also developed an online prediction server that can be easily used by experimental scientist and researchers to predict an unknown protein sequence as either insecticide-resistant or non-resistant protein. Moreover, computational identification of insecticide resistant proteins will supplement the efforts needed to develop insecticides in targeting the resistance proteins.

\section{Methods \\ Collection and processing of data}

In this study, protein sequences corresponding to four important groups of insecticide resistant genes viz., cytochrome P450, Kdr, Rdl and AChE were collected from insecticide resistance gene database (http://www.cib.re s.in/irgd/). We considered these four categories of genes because they represent important families of insecticide resistant genes which are resistant to commonly used insecticides. Besides, the resistant protein sequences were reported to be involved in two important resistance mechanisms viz., detoxification-based and target-based. Further, target-based resistant proteins are confined to three main targets of insecticides i.e., AChE, GABAgated chloride ion channel and voltage-gated sodium channel. A total of 822 sequences (772 cytochrome P450, $30 \mathrm{AChE}, 17 \mathrm{Rdl}$ and $3 \mathrm{Kdr}$ ) belonging to 11 
insect species (Additional file 1) were collected. Initially, we removed the sequences having non-standard residues. Then, four positive sets having 128, 285, 349 and 442 sequences were prepared, where the maximum pair-wise sequence identities were 40\%, 60\%, 70\% and 90\% respectively. The sequences with more than considered level of pair-wise sequence identity were removed using CDHIT [33]. For negative set, protein sequences (other than the positive sets) of the considered species were collected from the Uniprot (http://www.uniprot.org/) database. For the species Acyrthosiphon pisum and Tribolium castaneum, only the reviewed sequences were collected, as large number of sequence are present in Uniprot for these two species. On the other hand, all the sequences available for remaining nine species were collected. After removing the sequences having non-standard residues as well as the identical sequences, a total of 12613 sequences were obtained. Further, to avoid homologous bias in the negative dataset, sequences with $>40 \%$ pair-wise identity were removed using CDHIT. Finally a dataset with 3919 sequences was obtained and considered as the negative dataset.

\section{Feature generation}

Protein sequences are the strings of amino acid residues, and hence they need to be mapped onto numeric feature vectors before being used as input in machine learning classifier. In this study, amino acid composition (AAC), di-peptide composition (DPC), pseudo amino acid composition (PAAC), composition-transition-distribution (CTD) and auto correlation function (ACF) were used to transform the protein sequences into numeric feature vectors.

\section{Amino acid composition (AAC)}

$\mathrm{AAC}$ is a basic feature of protein sequence [34], which is closely associated with its attributes, such as sub-cellular location [35, 36], secondary structure content [37] and domain [38]. AAC consists of 20 discrete numbers, each of which represents the frequency of the native amino acids in a protein sequence. Based on the AAC, each protein sequence was encoded into a 20-dimensional numerical vector.

\section{Di-peptide composition (DPC)}

One of the limitations of AAC is that it does not take into account the local order information of amino acids in the protein. On the other hand, DPC, which gives a fixed pattern length of $400(20 \times 20)$, encapsulates the global information about each protein sequence and the order it contains [39]. For any di-peptide, its composition was computed as the ratio of the frequency of that di-peptide to the total number possible di-peptide in the protein sequence.

\section{Pseudo amino acid composition (PAAC)}

The concept of PAAC was originally introduced by Chou [40] for predicting the protein sub-cellular locations and membrane protein types. Based on the conventional AAC, Chou proposed a set of discrete numbers to take into account the sequence order effects. PAAC has been proven to be an extremely effective feature in many proteins and protein-related systems [41]. The PAAC for each protein sequence can be represented by a $(20+d)$-dimensional vector for $\mathrm{d}$-tier correlation factor. Here, the PAAC was extracted for $1^{\text {st }}$-tier correlation only, by which each sequence was transformed into a 21-dimensional numeric vector. For further details, one can refer to [40, 42, 43].

\section{Composition-transition-distribution (CTD)}

The CTD feature was introduced by Dubchak et al. [44] for predicting protein folding classes. Thereafter, the CTD feature has been adopted by many researchers for protein function and structure studies $[45,46]$. In CTD feature, composition $(C)$ is the number of amino acids of a particular type divided by the total number of amino acids. Transition (T) characterizes the frequency percentage with which amino acids of a particular type is followed by other amino acids. Distribution (D) measures the chain length within which the first 25\%, $50 \%, 75 \%$ and $100 \%$ of the amino acids of a particular type is located respectively. Based on the CTD feature, each protein sequence of length $L$ was encoded to a $L+\{L *(L-1) / 2\}+(L * 5)$-dimension numeric vector.

\section{Auto correlation function (ACF)}

Sequence autocorrelation-based features assume that the disturbances in each area are systematically related to those in adjacent areas [47]. This concept helps to analyze the dependency among the features of sequences in each location. Autocorrelation features were computed based on the distribution of amino acid properties along the sequence, using all the 531 amino acid indices available in AAindex database [48]. In this feature encoding, for an autocorrelation of order $n$, each sequence was transformed into a numeric vector of length $531 * n$.

\section{Supervised learning technique}

For classification purpose we used the support vector machine (SVM), which is a nonparametric algorithm developed by Vapnik [49]. It is a very promising and popular method for pattern recognition that has been widely used for prediction purpose in the field of bioinformatics [50-56]. It is proven to be very efficient in many biological analyses due to their ability to handle noise and large input dataset $[57,58]$. A brief description about the working principle of SVM is described as follows: 
Consider a binary classification problem with $N$ samples or input vectors $\mathbf{x}_{i} \in R^{d},(i=1,2, \ldots, N)$, where $\mathbf{x}_{i}$ with class levels $y_{i} \in\{-1,1\}$ can be considered as the $i^{\text {th }}$ protein or vector defined in $d$-dimensional space (which depends upon the sequence encoding approach). In present work, 1 refers to resistant class and -1 represents non-resistant class. The objective here is to construct a binary classifier from the available sample (training set) that has less probability of misclassifying future sample (test set). Non-linear SVM maps input vectors $\mathbf{x}_{i}{ }^{\prime} s$ into high dimensional feature space and constructs an optimal separating hyper-plane (OSH) that maximizes the distance between hyper-plane and nearest data points of each class in the space. Mathematically, the hyper-plane is represented as $y=\operatorname{sgn}\left(\mathbf{w}^{T} \mathbf{x}+b\right)$, where $\mathbf{w}$ represents a weight vector that can map training data in the input space to the outer space and $b$ represents bias. For a two class problem, it can be formulated as

$$
\left\{\begin{array}{l}
\mathbf{w}^{T} \mathbf{x}_{i}+b \geq 1 \text { if } y_{i}=1 \\
\mathbf{w}^{T} \mathbf{x}_{i}+b \leq-1 \text { if } y_{i}=-1
\end{array} .\right.
$$

The SVM training procedure involves optimization of convex quadratic problem i.e., with lagrangian multipliers $\alpha_{i} \geq 0$, maximize $\sum_{i=1}^{N} \alpha_{i}-\frac{1}{2} \sum_{i=1}^{N} \sum_{j=1}^{N} \alpha_{i} \alpha_{j} y_{i} y_{j} K\left(\mathbf{x}_{i} \mathbf{x}_{j}\right)$ subject to the constraints $0 \leq \alpha_{i} \leq c(i=1,2, \ldots, N)$ and $\sum_{i=1}^{N} \alpha_{i} y_{i}=0$ , where $c$ is the regularization parameter that controls trade-off between margin and classification error. The $\mathbf{x}_{j}{ }^{\prime}$ $s$ are called support vectors only if corresponding $\alpha_{j}>0$. After the SVM has been trained, the decision function for classification of query sequence $(\mathbf{x})$ can be formulated as

$$
f(\mathbf{x})=\operatorname{sgn}\left(\sum_{i=1}^{N} y_{i} \alpha_{i} K\left(\mathbf{x} \cdot \mathbf{x}_{i}\right)+b\right) .
$$

The choice of the proper kernel function $K$ is important to train SVM model because the power of SVM comes from the kernel representation that allows the nonlinear mapping of input space to a higher dimensional feature space. In this work, four commonly used kernel functions [59] viz., linear $\left(\mathbf{x}_{i} \mathbf{x}_{j}\right)$, polynomial $\left(\left(\gamma \mathbf{x}_{i}^{\prime} \mathbf{x}_{j}+r\right)^{d}\right)$, radial basis $\left(-\exp \left\{-\gamma\left\|\mathbf{x}_{i}-\mathbf{x}_{j}\right\|^{2}\right\}\right)$ and sigmoid $\left(\tanh \left(\gamma \mathbf{x}_{i} \mathbf{x}_{j}+r\right)\right)$ were used, where $r, d, \gamma>0$ are the kernel parameters.

\section{Validation of the model}

Cross-validation procedure has been widely accepted for assessing the performance of classifiers [60]. Thus, we used the 10-fold cross-validation to assess the performance of our approach. It was carried out by partitioning the dataset into 10 approximately equal-sized sets at random, where nine partitions were used to train the model and the remaining one part was used to assess the model accuracy. This process was repeated 10 times in such a way that each partition was tested once in the model.

\section{Performance evaluation}

Different performance metrics viz., sensitivity (Sn), specificity (Sp), accuracy (Ac), precision (Pre) and Matthew's correlation coefficient (MCC) were used to measure the accuracy of the developed prediction approach. The Sn, Sp, Ac, Pre and MCC parameters are defined as: $S n=t p /$ $(t p+f n), \quad S p=t n /(t n+f p), \quad A c=(t p+t n) /(t p+f n+t n+$ $f p), \quad$ Pre $=t p /(t p+f p), \quad M C C=[(t p \times t n)-(f p \times f n)] /$ $\sqrt{(t p+f n) \times(t p+f p) \times(t n+f n) \times(t n+f p)}$. True positive $(t p)$ is the number of resistant proteins correctly predicted as resistant proteins, true negative $(t n)$ is the number of non-resistant proteins correctly predicted as non-resistant proteins, false negative $(f n)$ is the number of resistant proteins incorrectly predicted as non-resistant proteins and false positive $(f p)$ is the number of non- resistant proteins incorrectly predicted as resistant proteins. Besides the above mentioned performance metrics, area under receiving operating characteristic curve (AUC-ROC) [61] was also used to measure the predictive ability. For given false positive rate $(\alpha)$ and true positive rate $(1-\beta)$ at different threshold values, the AUC-ROC was computed as $\sum_{i}\left\{\left(1-\beta_{i} \cdot \Delta \alpha\right)+(1 / 2)[\Delta(1-\beta) \cdot \Delta \alpha]\right\}$, where $\Delta(1-\beta)=\left(1-\beta_{i}\right)-\left(1-\beta_{i-1}\right), \quad \Delta \alpha=\alpha_{i}-\alpha_{i-1}$ and $i=1,2, \ldots, m$ (number of test instances) [62]. A subroutine in $\mathrm{R}$ programming language was written to compute the values of these performance metrics.

\section{Training and testing datasets}

Using four positive sets and one negative set (mentioned under "collection and processing of data"), four datasets were prepared that consists of both positive and negative sequences. Here each dataset contains a different positive set and the same negative set (3919 negative sequences). All the four datasets are highly unbalanced as the number sequences present in one class (non-resistant class) is much larger than the other class (resistant class). To avoid biasness towards the non-resistant class (major class) while predicting using machine learning classifier like SVM, balanced datasets were prepared that consists of same number of sequences from both the classes, where the sequences of the major class were randomly drawn from the available sequences of the major class. For instance, first balanced dataset contains 128 positive and 128 negative sequences, where the 128 negative sequences were randomly drawn from 3919 negative sequences. As the generalized predictive ability 
cannot be assured based on a single dataset, 100 sample sets were prepared, where each sample set consists of same number of positive and negative instances. Further, in each sample set, a 10-fold cross validation procedure was adopted. The performance metrics were computed by taking average over the 10 folds as well as over 100 sample sets.

\section{Mechanism-based classification}

The insecticide resistance mechanism can be broadly categorized into two types, viz., target-based mechanism and detoxification-based mechanism. The $\mathrm{Rdl}, \mathrm{Kdr}$ and $\mathrm{AChE}$ genes come under target-based and cytochrome P450 genes come under detoxification-based mechanism. To test whether the genes under these two categories are different or not, a binary classification was carried out by employing SVM, where 15 sequences (with $<90 \%$ pairwise sequence identity) from target-based and 452 sequences (with $<90 \%$ pair-wise sequence identity) from detoxification-based category were used. Similar to the classification of resistant and non-resistant proteins, 100 sample sets were prepared where each sample set consists of 15 sequences from each class. Since, there are 452 sequences in the detoxification-based category, 15 sequences were randomly drawn each time. As the number of sequences in each sample is not large, leave-one-out cross validation (LOOCV) technique was adopted for classification of detoxification- and target-based resistant proteins. Here, detoxification-based category was considered as positive class and target-based category as negative class.

\section{Comparison with blast algorithm}

Performance of the proposed approach was also compared with that of Blastp [63], PSI-Blast [64] and Delta-Blast [65], which are powerful algorithms to detect protein homologs. Further, comparison was made through 10-fold cross validation technique. For cross validation, offline (local) Blast software was used with blastp, psiblast and deltablast modules/programs in which the training set for each fold of cross validation was defined as the database and sequences of the corresponding test set were used as query. Each query sequence was predicted as the resistant or non-resistant category based on the top hit found in the blast search. Three different e-values i.e., $0.1,1$ and 10 were used to assess the performance of the Blastp, PSIBlast and Delta-Blast. Furthermore, performance of the proposed approach was compared based on best feature set with which higher accuracies were obtained as compared to the other feature sets.

\section{Performance evaluation using independent dataset}

To assess the generalized predictive ability of the proposed approach, its performance was further tested using an independent test dataset. The independent dataset was collected based on published literature that includes 53 cytochrome P450, $2 \mathrm{Kdr}, 3 \mathrm{Rdl}$ and $17 \mathrm{AChE}$ proteins. Specifically, 115 cytochrome $\mathrm{P} 450$ genes were reported by Hsu et al. [30]. Out of 115, we used 53 as they are available in NCBI. Similarly, $2 \mathrm{Kdr}, 3 \mathrm{Rdl}$ and $17 \mathrm{AChE}$ genes were collected from NCBI, based on the study of Zuo et al. [66], Wondji et al. [67] and $\mathrm{Li}$ and $\mathrm{Han}$ [68] respectively. Sequences of the independent test set are provided in Additional file 1.

\section{Development of prediction server}

An online prediction server was developed using HTML and PHP, where the combination of best feature set and classifier was used. A developed R-code was executed in background upon submission of the sequences in FASTA format to the server. The user has to submit the protein sequences having only standard amino acid residues. This server can be used to predict the likelihood of
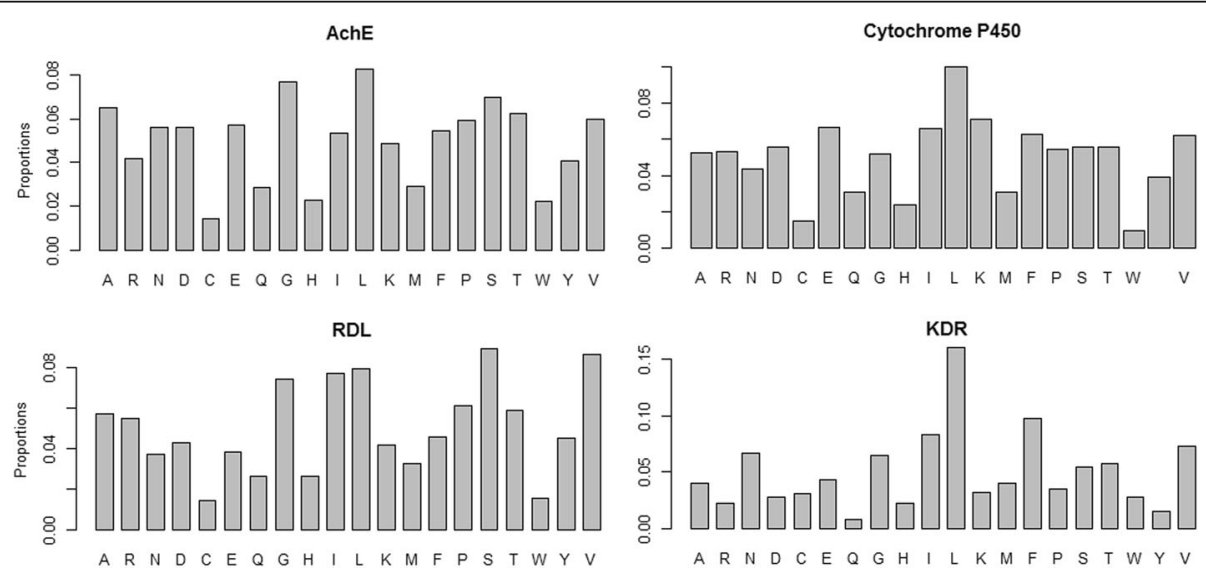

Fig. 1 Composition of amino acids in all the four categories of insecticide resistant proteins. It is observed that proportions of leucine are higher, whereas proportions of cystene and tryptophan are lower in all the four categories 


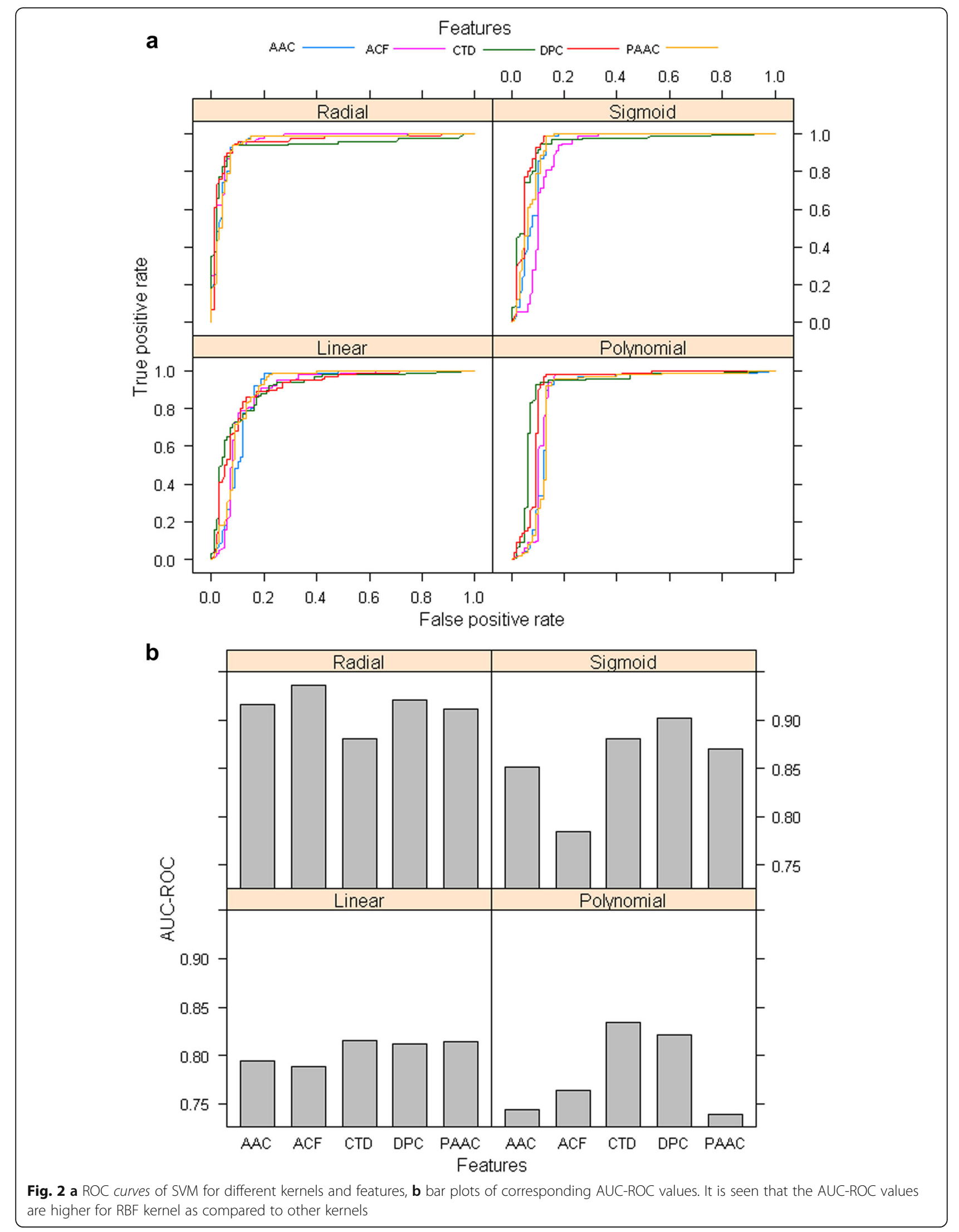


Table 1 Estimates of different performance metrics for SVM with RBF kernel in discriminating resistant from non-resistant proteins, under all the feature sets as well as different percentage of sequence identity in the positive dataset

\begin{tabular}{|c|c|c|c|c|c|c|c|}
\hline \multirow[b]{2}{*}{$\mathrm{Id}(\%)$} & \multirow[b]{2}{*}{ Feature } & \multicolumn{6}{|c|}{ Performance metrics } \\
\hline & & Sn & $\mathrm{Sp}$ & $A C$ & Pre & MCC & AUC-ROC \\
\hline \multirow[t]{5}{*}{40} & AAC & $0.836 \pm 0.018$ & $0.952 \pm 0.014$ & $0.894 \pm 0.012$ & $0.946 \pm 0.015$ & $0.794 \pm 0.024$ & $0.924 \pm 0.020$ \\
\hline & DPC & $0.849 \pm 0.013$ & $0.983 \pm 0.011$ & $0.916 \pm 0.009$ & $0.980 \pm 0.012$ & $0.839 \pm 0.017$ & $0.948 \pm 0.011$ \\
\hline & PAAC & $0.836 \pm 0.018$ & $0.956 \pm 0.014$ & $0.896 \pm 0.013$ & $0.951 \pm 0.015$ & $0.798 \pm 0.026$ & $0.922 \pm 0.018$ \\
\hline & CTD & $0.841 \pm 0.015$ & $0.981 \pm 0.011$ & $0.911 \pm 0.010$ & $0.978 \pm 0.013$ & $0.831 \pm 0.020$ & $0.932 \pm 0.010$ \\
\hline & $A C F$ & $0.836 \pm 0.017$ & 0.9530 .016 & $0.895 \pm 0.012$ & $0.947 \pm 0.017$ & $0.795 \pm 0.025$ & $0.901 \pm 0.017$ \\
\hline \multirow[t]{5}{*}{60} & AAC & $0.870 \pm 0.012$ & $0.959 \pm 0.008$ & $0.914 \pm 0.008$ & $0.955 \pm 0.009$ & $0.832 \pm 0.016$ & $0.946 \pm 0.008$ \\
\hline & DPC & $0.875 \pm 0.008$ & $0.986 \pm 0.007$ & $0.931 \pm 0.006$ & $0.984 \pm 0.007$ & $0.866 \pm 0.011$ & $0.972 \pm 0.005$ \\
\hline & PAAC & $0.870 \pm 0.014$ & $0.960 \pm 0.010$ & $0.915 \pm 0.010$ & $0.956 \pm 0.011$ & $0.833 \pm 0.020$ & $0.947 \pm 0.010$ \\
\hline & CTD & $0.860 \pm 0.011$ & $0.985 \pm 0.007$ & $0.923 \pm 0.007$ & $0.983 \pm 0.008$ & $0.852 \pm 0.014$ & $0.959 \pm 0.006$ \\
\hline & ACF & $0.869 \pm 0.011$ & $0.964 \pm 0.009$ & $0.917 \pm 0.007$ & $0.960 \pm 0.009$ & $0.837 \pm 0.015$ & $0.932 \pm 0.009$ \\
\hline \multirow[t]{5}{*}{70} & AAC & $0.886 \pm 0.011$ & $0.961 \pm 0.008$ & $0.924 \pm 0.008$ & $0.958 \pm 0.008$ & $0.850 \pm 0.015$ & $0.953 \pm 0.008$ \\
\hline & DPC & $0.883 \pm 0.008$ & $0.987 \pm 0.005$ & $0.935 \pm 0.005$ & $0.986 \pm 0.005$ & $0.875 \pm 0.009$ & $0.973 \pm 0.004$ \\
\hline & PAAC & $0.891 \pm 0.010$ & $0.961 \pm 0.008$ & $0.926 \pm 0.007$ & $0.958 \pm 0.008$ & $0.854 \pm 0.013$ & $0.955 \pm 0.007$ \\
\hline & CTD & $0.866 \pm 0.010$ & $0.987 \pm 0.005$ & $0.926 \pm 0.006$ & $0.985 \pm 0.006$ & $0.859 \pm 0.012$ & $0.961 \pm 0.006$ \\
\hline & ACF & $0.888 \pm 0.008$ & $0.963 \pm 0.009$ & $0.925 \pm 0.006$ & $0.960 \pm 0.009$ & $0.853 \pm 0.013$ & $0.948 \pm 0.007$ \\
\hline \multirow[t]{5}{*}{90} & AAC & $0.886 \pm 0.010$ & $0.959 \pm 0.006$ & $0.923 \pm 0.006$ & $0.956 \pm 0.006$ & $0.847 \pm 0.012$ & $0.955 \pm 0.006$ \\
\hline & DPC & $0.899 \pm 0.009$ & $0.989 \pm 0.005$ & $0.944 \pm 0.006$ & $0.988 \pm 0.005$ & $0.892 \pm 0.011$ & $0.978 \pm 0.004$ \\
\hline & PAAC & $0.889 \pm 0.011$ & $0.959 \pm 0.007$ & $0.924 \pm 0.007$ & $0.956 \pm 0.007$ & $0.850 \pm 0.014$ & $0.956 \pm 0.006$ \\
\hline & CTD & $0.887 \pm 0.008$ & $0.987 \pm 0.005$ & $0.937 \pm 0.005$ & $0.985 \pm 0.006$ & $0.878 \pm 0.010$ & $0.972 \pm 0.005$ \\
\hline & ACF & $0.894 \pm 0.010$ & $0.967 \pm 0.006$ & $0.930 \pm 0.006$ & $0.964 \pm 0.006$ & $0.863 \pm 0.013$ & $0.949 \pm 0.006$ \\
\hline
\end{tabular}

Id(\%): maximum percentage of pair-wise sequence identity present in the positive dataset

Sn Sensitivity, Sp Specificity, Ac Accuracy, Pre Precision, MCC Matthew's correlation coefficient, AUC-ROC area under ROC curves

any unknown protein sequence being an insecticide resistant protein with certain probability.

\section{Results}

\section{Analysis of amino acid compositions}

The composition of amino acids in four different groups of insecticide resistant proteins is shown in Fig. 1. It is seen that the proportions of leucine (L) are higher, whereas the proportions of cystene $(C)$ and tryptophan (W) are lower in all the four categories.

\section{Analysis of kernel functions}

Based on a sample dataset consisting of 100 positive and 100 negative sequences that were drawn randomly from the available positive and negative sequences, performance of SVM was analyzed. ROC curves for all the four

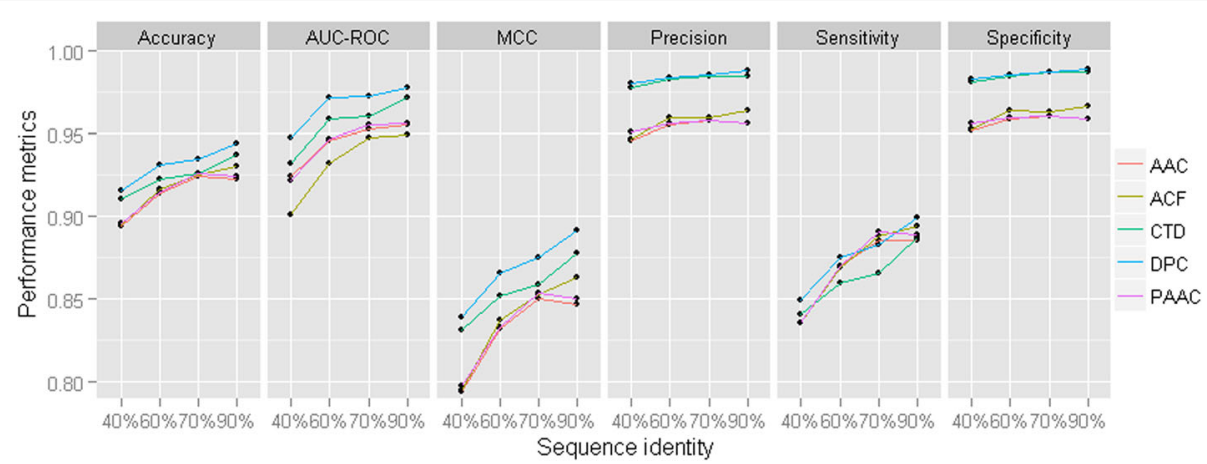

Fig. 3 Performance metrics of SVM with RBF kernel for different feature sets and different percentage of pair-wise sequence identity in the positive set. It can be seen that the performance metrics are higher for DPC feature set as compared to other feature sets, irrespective of the percentage of sequence identity in the positive dataset 
Table 2 Estimates of performance metrics for classification of detoxification and target-based resistant proteins, under different feature sets

\begin{tabular}{lllllll}
\hline Feature & Sn & Sp & AC & Pre & MCC & AUC-ROC \\
\hline AAC & $0.927 \pm 0.020$ & $0.966 \pm 0.042$ & $0.946 \pm 0.024$ & $0.966 \pm 0.041$ & $0.894 \pm 0.049$ & $0.960 \pm 0.023$ \\
DPC & $0.967 \pm 0.067$ & $0.985 \pm 0.031$ & $0.976 \pm 0.035$ & $0.986 \pm 0.029$ & $0.955 \pm 0.065$ & $0.972 \pm 0.051$ \\
PAAC & $0.929 \pm 0.016$ & $0.952 \pm 0.048$ & $0.941 \pm 0.027$ & $0.953 \pm 0.046$ & $0.883 \pm 0.054$ & $0.956 \pm 0.028$ \\
CTD & $0.895 \pm 0.042$ & $0.979 \pm 0.035$ & $0.937 \pm 0.024$ & $0.979 \pm 0.035$ & $0.879 \pm 0.047$ & $0.935 \pm 0.036$ \\
ACF & $0.912 \pm 0.041$ & $0.927 \pm 0.051$ & $0.919 \pm 0.037$ & $0.927 \pm 0.049$ & $0.840 \pm 0.074$ & $0.967 \pm 0.021$ \\
\hline
\end{tabular}

Sn Sensitivity, Sp Specificity, Ac Accuracy, Pre Precision, MCC Matthew's correlation coefficient, AUC-ROC area under ROC curves

kernels as well as for all the five feature sets are shown in Fig. 2a and the corresponding AUC-ROC values are shown in bar plots (Fig. 2b). From the ROC curves it is not clear that which kernel is better, whereas from AUC-ROC plots it is clear that the values of AUC-ROC are higher for the RBF kernel, irrespective of the feature set used. Though in RBF kernel the AUC-ROC for ACF feature set is highest, it is difficult to choose the best feature set while other three kernels are taken into account. Therefore, all the feature sets and the RBF kernel were used for further analysis.

\section{Cross-validation performance analysis}

For all the four datasets (mentioned under "Training and testing datasets") as well as for all the feature sets, performance metrics averaged over 10 -fold as well as 100 sample sets are given in Table 1. Moreover, to analyze the trend in accuracies, performance metrics are also plotted in line graphs (Fig. 3). It is observed that the sensitivities are less as compared to the specificities (Table 1). Further, higher accuracies are observed for the dataset having resistant proteins with $<90 \%$ pair-wise sequence identity, whereas lower accuracies are observed for the dataset having resistant proteins with $<40 \%$ pairwise sequence identity (Table 1 and Fig. 3). Though the specificities are observed almost unchanged, sensitivities are observed to be increased with increase in the percentage of pair-wise sequence identity in the positive dataset (Fig. 3). Besides, it is seen that the most of the performance metrics for DPC and CTD feature sets are higher as compared to the other feature sets (AAC, PAAC and ACF). In particular, overall accuracy ( 90\%), MCC ( 89\%) and AUC-ROC ( 98\%) are observed to be highest for DPC feature set. Since the number of sequences in the positive dataset having sequences with $<90 \%$ pair-wise sequence identity is larger as compared to the dataset having sequences with $<40 \%$ pair-wise sequence identity, the former one is used in subsequent analyses.

\section{Analysis of mechanism-based classification}

The values of performance metrics, with regard to classification of resistant proteins involved in target-based mechanism and detoxification-based mechanism, measured over LOOCV as well as 100 sample sets are given in Table 2. Performance metrics for all the feature sets are observed $\geq 90 \%$ and are found to be highest in case of DPC feature set. More specifically, overall accuracy for the DPC feature set is observed $>97 \%$, with $>95 \%$ MCC and $>97 \%$ AUC-ROC. Though the number of features for AAC and PAAC feature sets are almost same, classification accuracies for AAC feature set are seen to be higher than that of PAAC feature set. Since the sensitivity and specificity are $>90 \%$, it is inferred that hardly one sequence is misclassified in each category (as the number of sequences in each category is only 15 ).

\section{Discriminating target-based resistant proteins from non-resistant proteins}

With regard to classification of target-based resistant proteins and non-resistant proteins, performance metrics over LOOCV and 100 sample sets (where each sample set consists of 15 target-based resistant proteins and 15 nonresistant proteins that were randomly drawn from the 3919 non-resistant proteins) are given in Table 3. The values of performance metrics are observed to be higher

Table 3 Estimates of performance metrics for discriminating target-based resistant proteins from non-resistant proteins, under different features

\begin{tabular}{lllllll}
\hline Feature & Sn & Sp & AC & Pre & MCC & AUC-ROC \\
\hline AAC & $0.912 \pm 0.031$ & $0.940 \pm 0.055$ & $0.926 \pm 0.034$ & $0.941 \pm 0.052$ & $0.854 \pm 0.068$ & $0.879 \pm 0.045$ \\
DPC & $0.924 \pm 0.090$ & $0.981 \pm 0.041$ & $0.952 \pm 0.057$ & $0.979 \pm 0.043$ & $0.909 \pm 0.111$ & $0.924 \pm 0.083$ \\
PAAC & $0.919 \pm 0.029$ & $0.947 \pm 0.053$ & $0.933 \pm 0.034$ & $0.948 \pm 0.051$ & $0.868 \pm 0.067$ & $0.880 \pm 0.043$ \\
CTD & $0.855 \pm 0.037$ & $0.945 \pm 0.047$ & $0.900 \pm 0.034$ & $0.941 \pm 0.049$ & $0.804 \pm 0.069$ & $0.844 \pm 0.028$ \\
ACF & $0.915 \pm 0.037$ & $0.927 \pm 0.054$ & $0.921 \pm 0.037$ & $0.928 \pm 0.051$ & $0.844 \pm 0.074$ & $0.846 \pm 0.043$
\end{tabular}

Sn Sensitivity, Sp Specificity, Ac Accuracy, Pre Precision, MCC Matthew's correlation coefficient, AUC-ROC area under ROC curves 
Table 4 Estimates of different performance metrics for discriminating detoxification-based resistant proteins from non-resistant proteins

\begin{tabular}{lllllll}
\hline Feature & Sn & Sp & AC & Pre & MCC & AUC-ROC \\
\hline AAC & $0.898 \pm 0.009$ & $0.963 \pm 0.006$ & $0.931 \pm 0.006$ & $0.960 \pm 0.007$ & $0.863 \pm 0.013$ & $0.960 \pm 0.007$ \\
DPC & $0.911 \pm 0.006$ & $0.992 \pm 0.004$ & $0.951 \pm 0.004$ & $0.991 \pm 0.004$ & $0.905 \pm 0.008$ & $0.980 \pm 0.004$ \\
PAAC & $0.901 \pm 0.008$ & $0.965 \pm 0.006$ & $0.933 \pm 0.006$ & $0.962 \pm 0.007$ & $0.867 \pm 0.012$ & $0.960 \pm 0.006$ \\
CTD & $0.907 \pm 0.007$ & $0.990 \pm 0.004$ & $0.948 \pm 0.005$ & $0.989 \pm 0.004$ & $0.900 \pm 0.009$ & $0.974 \pm 0.004$ \\
ACF & $0.912 \pm 0.007$ & $0.969 \pm 0.006$ & $0.941 \pm 0.005$ & $0.968 \pm 0.006$ & $0.883 \pm 0.010$ & $0.959 \pm 0.005$ \\
\hline
\end{tabular}

Sn Sensitivity, Sp Specificity, Ac Accuracy, Pre Precision, MCC Matthew's correlation coefficient, AUC-ROC area under ROC curves

for DPC feature set. Specifically, accuracies in terms of all the performance metrics are observed $\geq 90 \%$ for DPC feature set, whereas the values of MCC and AUC-ROC for rest of the feature sets are observed to be $<90 \%$.

\section{Discriminating detoxification-based resistant proteins from non-resistant proteins}

The classification was also made between 452 detoxificationbased resistant proteins and 3919 non-resistant proteins, by using SVM with RBF kernel. Performances metrics were computed over 10 folds of cross validation as well as 100 sample sets (where each sample consists of 452 detoxification-based resistant proteins and 452 nonresistant proteins that were drawn randomly from the 3919 non-resistant proteins) are presented in Table 4. It is observed that the accuracies are higher for DPC feature set and lower for AAC feature set. In particular, the values of all the performances metrics for both CTD and DPC feature sets are $\geq 90 \%$ (Table 4 ). Barring sensitivity, the values of performance metrics in discriminating the detoxification-based resistant proteins from non-resistant proteins (Table 4) are higher as compared to that of discriminating target-based resistant proteins from nonresistant proteins (Table 3).

\section{Comparative analysis}

For comparing the proposed approach with Blast algorithms, we prepared two different datasets. The first dataset contains 442 resistant proteins (with $<90 \%$ pair-wise sequence identity) and randomly drawn 442 non-resistant proteins (with $<40 \%$ pair-wise sequence identity), and the second dataset contains 128 resistant proteins (with $<40 \%$ pair-wise sequence identity) and randomly drawn 128 non-resistant proteins (with $<40 \%$ pair-wise sequence identity). Furthermore, performance of the proposed approach was compared based on DPC feature set only as higher accuracies were obtained for this feature set as compared to the other feature sets. In both the datasets, no hits were found for most of the query sequences with e-values 0.1 and 1. However, hits were found for all the query sequences with e-value 10. Therefore, comparison was made based on e-value 10 only, and the accuracies averaged over 10 -folds are given in Table 5 . It is observed that the overall accuracies of the proposed approach are $\sim 10 \%$ higher than that of Blastp, PSIBlast and Delta-Blast, in both datasets (Table 5). Though, true positive rates (sensitivity) of the Blast algorithms are higher, false positive rates (specificity) are much lower at the same time. Among the Blast algorithms, Delta-Blast performed better than both Blastp and PSI-Blast, with both the datasets (Table 5). Barring sensitivity, the proposed approach performed better than Blast algorithms in terms of all the performance metrics. It is further seen that the specificities are higher for the first dataset as compared to the second dataset.

\section{Performance analysis based on independent test dataset}

Both the datasets mentioned in "comparative analysis" section were used to train the model for prediction of the level (as resistant or non-resistant) of each test sequence. Furthermore, none of the test sequences were present in the training set. It is observed that 69 out of 75 are correctly predicted while first dataset is used as training set (Table 6). On the other hand, all the 75 instances are correctly identified as insecticide resistant proteins with second dataset as training set (Table 6). Besides, it is seen that most of the sequences are correctly predicted with $>0.9$ probabilities irrespective of

Table 5 Performance metrics for the proposed approach, Blast, PSI-Blast and Delta-Blast, in discriminating the resistant proteins from non-resistant proteins, where the positive dataset consists of $<40 \%$ (first) and $<90 \%$ (second) pair-wise sequence identity

\begin{tabular}{lllllll}
\hline Dataset & Method & Sn & Sp & Ac & Pre & MCC \\
\hline First & Proposed & 0.897 & 0.934 & 0.916 & 0.933 & 0.836 \\
& Blast & 0.961 & 0.611 & 0.786 & 0.713 & 0.617 \\
& PSI-Blast & 0.959 & 0.602 & 0.780 & 0.707 & 0.607 \\
& Delta-Blast & 0.961 & 0.652 & 0.806 & 0.735 & 0.647 \\
Second & Proposed & 0.875 & 0.891 & 0.883 & 0.901 & 0.784 \\
& Blast & 0.958 & 0.350 & 0.654 & 0.596 & 0.392 \\
& PSI-Blast & 0.958 & 0.358 & 0.658 & 0.601 & 0.400 \\
& Delta-Blast & 0.958 & 0.466 & 0.712 & 0.646 & 0.495 \\
\hline
\end{tabular}

Here, AUC-ROC values were not computed, as in Blast algorithms accuracies are computed based on number of hits

Sn Sensitivity, Sp Specificity, Ac Accuracy, Pre Precision, MCC Matthew's correlation coefficient 
Table 6 Performance of the proposed approach based on an independent dataset of 75 insecticide resistant proteins

\begin{tabular}{lllll}
\hline & & & Predicted \\
\cline { 1 - 2 } \cline { 5 - 5 } Resistance family & Observed & & $1^{\text {st }}$ training model & $2^{\text {nd }}$ training model \\
\hline Cytochrome P450 & 53 & 51 & 53 \\
Kdr & 2 & 2 & 2 \\
Rdl & 3 & 3 & 3 \\
AChE & 17 & 13 & 17 \\
Total & 75 & 69 & 75 \\
\hline
\end{tabular}

the training datasets (Fig. 4). More clearly, 2 test sequences of cytochrome P450 and 4 sequences of AChE are misclassified in the first training dataset (Fig. 4).

\section{Online prediction server: DIRProt}

A web server DIRProt has been developed to discriminate the insecticide resistant proteins from non-resistant proteins. This server has been trained with the SVM (with RBF kernel) for prediction of insecticide resistant proteins based on DPC feature set. The web pages showing the execution and results for an example dataset are shown in Fig. 5a and b respectively. Help pages are also provided to guide the user regarding generation of features, prediction method and input-output. The sequences in FASTA format along with the annotations and probabilities with which they are predicted as resistance proteins are shown in the result page. For reproducible research, the trained datasets are also provided in the server. The prediction server is made freely accessible at http://cabgri d.res.in:8080/dirprot for academic users.

\section{Discussion}

Extensive use of chemical insecticides has been selecting resistant population of insect species to different insecticides, worldwide [69, 70]. Around 590 insect species have been reported to resist different insecticides till the end of 2014 [71]. Insecticidal resistance has been associated with the genetic changes in insects. For instance, a mutation in an insect can alter the behavior, metabolism and physiology by which insect may gain advantage in resisting to different insecticides [70]. Most of the earlier studies are dealt with the mutational changes associated with the insecticide resistance. Though insecticide resistance is an important researchable issue, there is no computational tool available for prediction of insecticide resistant proteins. Therefore, we made an attempt to present the first computational approach for prediction of insecticide resistant proteins.

We considered four different categories of insecticide resistant proteins corresponding to four different classes of insecticide resistance genes viz., cytochrome P450, AChE, Rdl and Kdr. The leucine content was predominantly found in all the four categories of proteins, which has been reported to play an important role in insecticide resistance. For instance, Prince et al. [72] reported that leucine-rich repeat receptor-like kinase "brassinosteroid insensitive1-associated kinase1" contributes to the innate immunity to aphids in Arabidopsis. The valine to leucine (V419L) and the leucine to isoleucine mutations (L925I) were identified in three pesticide-resistant strains of bed bug (Cimex lectularius) [73]. Further, the composition of tryptophan which has been reported to present in the active site that interacts with trimethylammonium cationic group of AchE was found lowest [73]. Hassani et al. [74] described that lysine and tryptophan (Lys12 and Trp39 and Trp54) are the most reactive residues that play important role in disrupting the function of neuronal sodium channels by Ts gamma, which is the most potent neurotoxin in the venom of the Brazilian scorpion Tityus serrulatus.

For classification of insecticide resistant and nonresistant proteins, initially the sequences were transformed into numeric feature vectors based on different feature generation techniques viz., AAC, DPC, PAAC, $A C F$ and CTD. The encoded numeric vectors were then

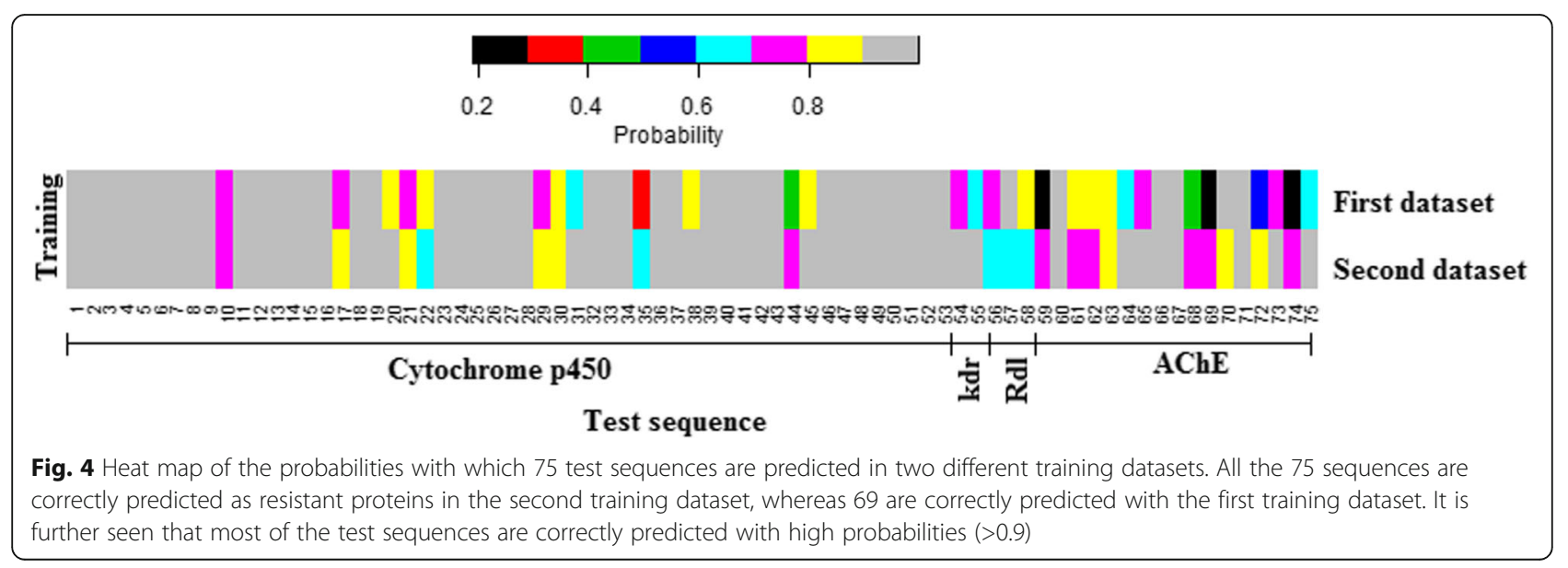




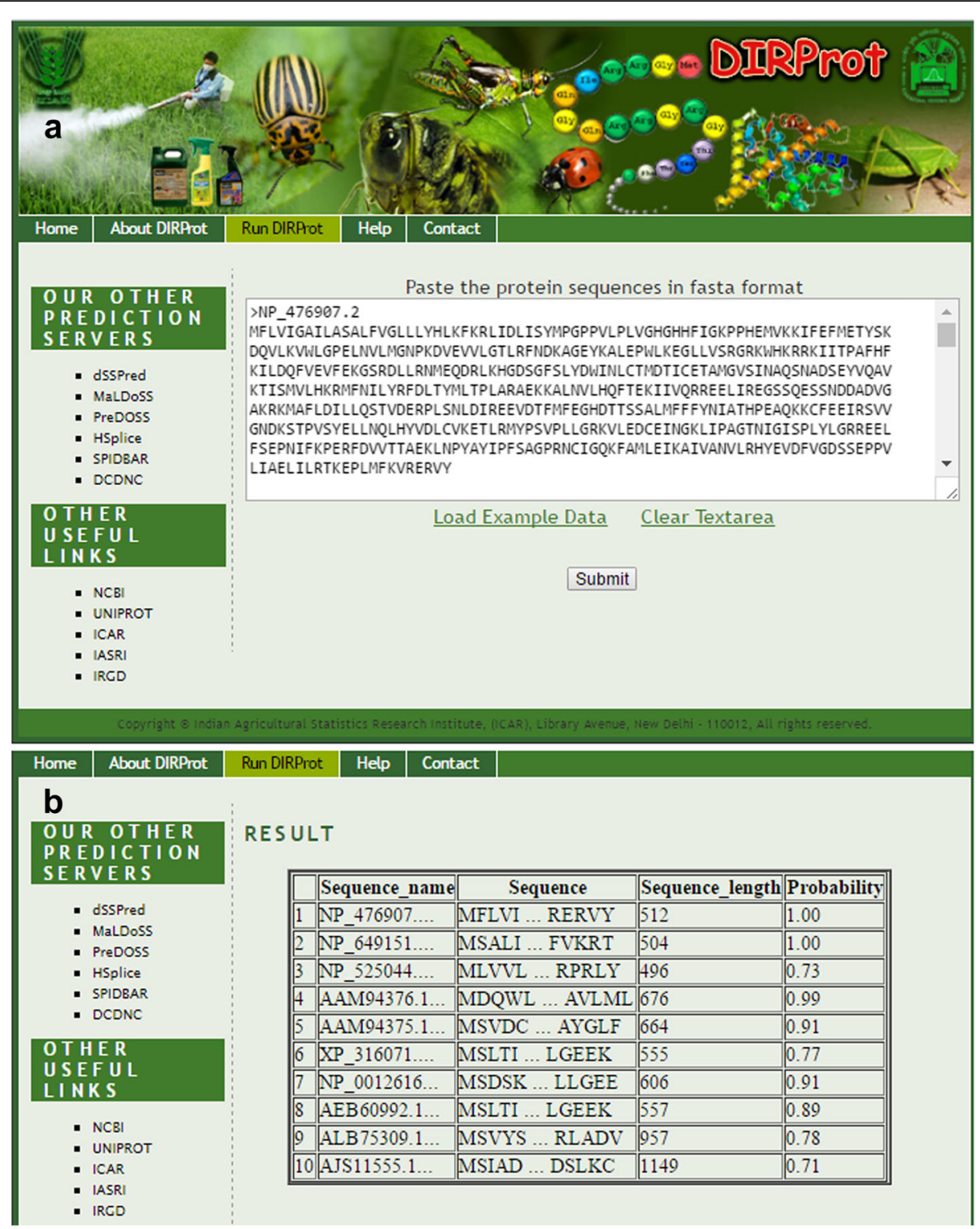

Fig. 5 a Server page of DIRProt, $\mathbf{b}$ result page after execution with an example dataset. The result page is displayed in a tabular form, where the last column is the probabilities with which the each sequences are predicted as insecticide-resistant proteins

used as input in binary SVM classifier. Prediction accuracies were found to be higher for RBF kernel as compared to the other three kernels of SVM. Further, the classification accuracies were found higher for DPC feature set as compared to the other feature sets, which may be due to the fact that in DPC the local ordering of amino acids were taken into account [42, 43]. Furthermore, in cross validation analysis (Table 1 ), the sensitivity was found to be increased with increase in the percentage of pair-wise sequence identity in the positive dataset. This may be due to the fact that with increase in the pair-wise sequence identity in the positive dataset, it is less-likely that a positive sequence will be misclassified in the negative dataset. The accuracy in discriminating the target-based and detoxification-based resistance proteins from non-resistant proteins was also found to be higher. Besides, higher discrimination accuracy was also observed between target-based and detoxification-based resistance proteins. Thus, it can be inferred that the composition of di-peptides are not only different between resistant and non-resistant proteins but also among insecticide resistant proteins involved in different insecticide resistance mechanisms.

The performance of the proposed approach was compared with Blast, PSI-Blast and Delta-Blast algorithms. Though, prediction was made for three e-values i.e., 0.1, 1 and 10, no hits were found for most of the query sequences (particularly negative) for the first two e- 
values. Thus performance metrics were computed based on e-value 10 only, which is also the default e-value in Blast algorithms. Though the resistant proteins were predicted with higher accuracy, the specificities were found much lower. It was also found that the specificities are higher for the first dataset as compared to the second dataset. One of the possible reasons for this may be that when the pair-wise sequence identity is $<40 \%$ in the positive class (first dataset), sequence similarity between the classes will be less. On the other hand, when the pair-wise sequence identity is $<90 \%$ in the positive class, sequence similarity between the positive and negative classes will be more by which the likelihood of a sequence of the negative class to be predicted in the positive class will be more and vice versa. In terms of overall accuracy, the proposed approach outperformed all the three variations of Blast algorithm. Among the Blast algorithms, Delta-Blast performed better followed by PSI-Blast and Blast. The performance of the proposed approach was also assessed using an independent test dataset consisting of 75 resistant protein sequences (53 cytochrome P450, $2 \mathrm{Kdr}, 3 \mathrm{Rdl}$ and $17 \mathrm{AChE}$ ). Out these 75 sequences, all were correctly predicted when the pairwise sequence identity was $<90 \%$ in the positive dataset of training set, whereas 69 were correctly predicted in for the training dataset having positive sequences with $<40 \%$ pair-wise sequence identity. Nevertheless, the proposed approach achieved higher accuracy for predicting the insecticide resistant proteins.

\section{Conclusions}

This paper presents the first computational approach for predicting the insecticide resistant proteins. Based on this approach, a web server has also been developed that can be easily used by the scientists and researchers to computationally identify the insecticide resistant proteins. The proposed computational approach is believed to supplement the wet-lab experiments for identifying and targeting the insecticide resistant proteins to develop dynamic and efficient insecticides.

\section{Additional file}

Additional file 1: It contains the list of insect species and corresponding insecticide resistant gene types that were used in this study. This file also contains the 75 insecticide resistant protein sequences that were used as independent dataset. (PDF $185 \mathrm{~kb}$ )

\section{Abbreviations}

AAC: Amino acid composition; Ac: Accuracy; ACF: Auto correlation function; AChE: Acetylcholinesterases; AUC-ROC: Area under ROC curves; CTD: Composition-trainsition-distribution; DPC: Dipeptide composition; FN: False negative; FP: False positive; GABA: $\gamma$-amino butyric acid; GSTs: Glutathione S-transferases; Kdr: Knock down resistance; MCC: Matthew's correlation coefficient; OSH: Optimal separating hyper-plane; PAAC: Pseudo amino acid composition; Pre: Precision; RBF: Radial basis function;
Rdl: Resistance to dieldrin; ROC: Receiving operating characteristics; Sn: Sensitivity; Sp: Specificity; SVM: Support vector machine; TN: True negative; TP: True positive

\section{Acknowledgements}

Authors are grateful to the reviewers for their constructive suggestions that lead to significant improvement of the manuscript. Authors also acknowledge Supriya Puram and Nalini Kanta Choudhury for their help in data processing

\section{Funding}

The research is supported by the grant (Agril.Edn.4-1/2013-A\&P dated 11.11 .2014 ) received from Indian Council of Agriculture Research (ICAR) for Centre for Agricultural Bioinformatics (CABin) scheme of Indian Agricultural Statistics Research Institute (IASRI). The funding body played no role in design or conclusion of this study.

\section{Availability of data and materials}

The insecticide resistant proteins were collected from insecticide resistant gene database available at http://www.cib.res.in/irgd/. The sequences of the negative dataset were collected from UniProt database available at http:// www.uniprot.org/. These datasets were also provided in the developed DIRProt server (http://cabgrid.res.in:8080/dirprot/), where the 442 insecticide resistant proteins are available at http://cabgrid.res.in:8080/dirprot/ resist_protein.txt and 440 non-resistant proteins are available at http:// cabgrid.res.in:8080/dirprot/notresist_protein.txt.

\section{Authors' contributions}

Conceived and designed the study: PKM, ARR; Collected and analyzed the sequence dataset: TKS, AB, PKM; Developed the prediction approach: PKM; Developed the web server: TKS, PKM; Drafted the manuscript: PKM, AB, TKS Corrected and refined the manuscript: PKM, TKS, ARR; All authors read and approved the final manuscript.

\section{Competing interests}

The authors' declared that they have no competing interest.

Consent for publication

Not applicable.

Ethics approval and consent to participate Not applicable.

\section{Publisher's Note}

Springer Nature remains neutral with regard to jurisdictional claims in published maps and institutional affiliations.

\section{Author details}

${ }^{1}$ Division of Statistical Genetics, ICAR-Indian Agricultural Statistics Research Institute, New Delhi 110012, India. ${ }^{2}$ Centre for Agricultural Bioinformatics, ICAR-Indian Agricultural Statistics Research Institute, New Delhi 110012, India. ${ }^{3}$ Department of Bioinformatics, Janta Vedic College, BarautBaghpat, 250611, Uttar Pradesh, India.

Received: 17 November 2016 Accepted: 9 March 2017

Published online: 24 March 2017

References

1. Insecticide Resistance Action Committee (IRAC). 48th Meeting IRAC International. 2013. http://www.irac-online.org.

2. Zhu F, Liu N. Differential expression of CYP6A5 and CYP6A5V2 in pyrethroidresistant house flies, Musca domestica. Arch Insect Biochem Physiol. 2008; 34:147-61.

3. Zhu F, Li T, Zhang L, Liu N. Co-up-regulation of three P450 genes in response to permethrin exposure in permethrin resistant house flies, Musca domestica. BMC Physiology. 2008:8:18.

4. Liu N, Li T, Reid WR, Yang T, Zhang L. Multiple cytochrome P450 genes: their constitutive overexpression and permethrin induction in insecticide resistant mosquitoes, Culex quinquefasciatus. Plos one. 2011;6:e23403. 
5. Liu N. In: Perveen F, editor. Pyrethroid resistance in insects: genes, mechanisms, and regulation, insecticides - advances in integrated pest management. 2012. p. 457-68.

6. Vontas JG, Small GJ, Hemingway J. Glutathione S-transferases as antioxidant defence agents confer pyrethroid resistance in Nilaparvata lugens. Biochem J. 2001;357:65-72.

7. Oakeshott JG, Horne I, Sutherland TD, Russell RJ. The genomics of insecticide resistance. Genome Biol. 2003:4:202.

8. Jackson CJ, Oakeshott JG, Sanchez-Hernandez JC, Wheelock CE. Carboxylesterases in the Metabolism and Toxicity of Pesticides. In: Satoh T, Gupta RC, eds. Anticholinesterase Pesticides. Hoboken: John Wiley \& Sons, Inc; 2012. p. 57-75.

9. Hemingway J, Hawkes NJ, McCarroll L, Ranson H. The molecular basis of insecticide resistance in mosquitoes. Insect Biochem Mol Biol. 2004;34(7): 653-65.

10. Li XC, Schuler MA, Berenbaum MR. Molecular mechanisms of metabolic resistance to synthetic and natural xenobiotics. Annu Rev Entomol. 2007; $52: 231-53$.

11. Narahashi T. Molecular and cellular approaches to neurotoxicology: past, present and future. In: Lunt GG, editor. Neurotox '88: molecular basis of drug and pesticide action. New York: Elsevier; 1988. p. 563-82.

12. Brogdon WG, MCAllister JC. Insecticide resistance and vector control. Emerg Infect Diseases. 1998:4(4):605-13.

13. Ahmad M, Denholm I, Bromilow RH. Delayed cuticular penetration and enhanced metabolism of deltamethrin in pyrethroid-resistant strains of Helicoverpa armigera from China and Pakistan. Pest Manag Sci. 2006;62:805-10.

14. Zhang J, Goyer C, Pelletier Y. Environmental stresses induce the expression of putative glycine-rich insect cuticular protein genes in adult Leptinotarsa decemlineata (Say). Insect Mol Biol. 2008;17:209-16.

15. Feyereisen R. Insect cytochrome P450. In: Gilbert LI, latrou K, Gill S, editors. Comprehensive molecular insect science, vol. 4. Oxford: Elsevier; 2005. p. 1-77.

16. Komagata O, Kasai S, Tomita T. Overexpression of cytochrome P450 genes in pyrethroid-resistant Culex quinquefasciatus. Insect Biochem Mol Biol. 2010; 40:146-52.

17. Ranson H, Hemingway J. Glutathione transferases. In: Gilbert LI, latrou K, Gill S, editors. Comprehensive molecular insect science, vol. 5. Oxford: Elsevier; 2005. p. 383-402.

18. Vontas J, Blass C, Koutsos AC, David JP, Kafatos FC, Louis C, Hemingway J, Christophides GK, Ranson H. Gene expression in insecticide resistant and susceptible Anopheles gambiae strains constitutively or after insecticide exposure. Insect Mol Biol. 2005;14:509-21.

19. Ffrench-Constant RH, Daborn PJ, Le Goff G. The genetics and genomics of insecticide resistance. Trends Genet. 2004;20(3):163-70.

20. Ffrench-Constant RH, Anthony N, Aronstein K, Rocheleau T, Stilwell G. Cyclodiene insecticide resistance: from molecular to population genetics. Annu Rev Entomol. 2000;45:449-66

21. Ffrench-Constant R, Rocheleau TA, Steichen JC, Chalmers AE. A point mutation in a Drosophila GABA receptor confers insecticide resistance. Nature. 1993:363(6428):449-51.

22. Donnelly MJ, Corbel V, Weetman D, Wilding CS, Williamson MS, Black WCT. Does kdr genotype predict insecticide-resistance phenotype in mosquitoes? Trends Parasitol. 2009;25:213-9

23. Williamson MS, Denholm I, Bell CA, Devonshire AL. Knockdown resistance $(k d r)$ to DDT and pyrethroid insecticides maps to a sodium channel gene locus in the housefly (Musca domestica). Mol Gen Genet. 1993:240:17-22.

24. Miyazaki M, Ohyama K, Dunlap DY, Matsumura F. Cloning and sequencing of the para-type sodium channel gene from susceptible and kdr-resistance German cockroaches (Blatella germanica) and the house fly (Musca domestica). Mol Gen Genet. 1996;252:61-8.

25. Davies TE, O'Reilly AO, Field LM, Wallace B, Williamson MS. Knockdown resistance to DDT and pyrethroids: from target-site mutations to molecular modelling. Pest Manag Sci. 2008;64(11):1126-30.

26. Mutero A, Pralavorio M, Bride JM, Fournier D. Resistance-associated point mutations in insecticide-insensitive acetylcholinesterase. Proc Natl Acad Sci U S A. 1994;91:5922-6

27. Ffrench-Constant $\mathrm{RH}$. The molecular genetics of insecticide resistance. Genetics. 2013;194:807-15.

28. Riveron JM, Yunta C, Ibrahim SS, Djouaka R, Irving H, Menze BD, Ismail HM Hemingway J, Ranson H, Albert A, Wondji CS. A single mutation in the GSTe2 gene allows tracking of metabolically based insecticide resistance in a major malaria vector. Genome Biol. 2014;15:R27.
29. Nwane P, Etang J, Chouaïbou M, Toto JC, Mimpfoundi R, Simard F. Kdrbased insecticide resistance in Anopheles gambiae s.s populations in Cameroon: spread of the L1014F and L1014S mutations. BMC Res Notes. 2011:4:463.

30. Hsu JC, Chien TY, Hu CC, Chen MJM, Wu WJ, Feng HT, Haymer DS, Chen CY. Discovery of genes related to insecticide resistance in Bactrocera dorsalis by functional genomic analysis of a De Novo assembled transcriptome. PLoS ONE. 2012;7(8):e40950.

31. Dou W, Shen GM, Niu JZ, Ding TB, Wei DD, Wang JJ. Mining genes involved in insecticide resistance of Liposcelis bostrychophila badonnel by transcriptome and expression profile analysis. PLoS ONE. 2013;8(11):e79878.

32. Cui L, Rui C, Yang D, Wang Z, Yuan H. De novo transcriptome and expression profile analyses of the Asian corn borer (Ostrinia furnacalis) reveals relevant flubendiamide response genes. BMC Genomics. 2017;18:20.

33. Huang Y, Niu B, Gao Y, Fu L, Li W. CD-HIT suite: a web server for clustering and comparing biological sequences. Bioinform. 2010;26:680-2.

34. Cai YD, Chou KC. Predicting membrane protein type by functional domain composition and pseudo-amino acid composition. J Theor Biol. 2006;238(2): 395-400.

35. Hoglund A, Donnes P, Blum T, Adolph HW, Kohlbacher O. MultiLoc: prediction of protein subcellular localization using N-terminal targeting sequences, sequence motifs and amino acid composition. Bioinformatics. 2006;22:1158-65.

36. Tamura T, Akutsu T. Subcellular location prediction of proteins using support vector machines with alignment of block sequences utilizing amino acid composition. BMC Bioinformatics. 2007:8:466.

37. Lee $\mathrm{S}$, Lee BC, Kim D. Prediction of protein secondary structure content using amino acid composition and evolutionary information. Proteins. 2006; 62:1107-14

38. Dumontier M, Yao R, Feldman HJ, Hogue CW. Armadillo: domain boundary prediction by amino acid composition. J Mol Biol. 2005;350:1061-73.

39. Ding Y, Cai $Y$, Zhang G, Xu W. The influence of dipeptide composition on protein hermostability. FEBS Lett. 2004;569:284-8.

40. Chou KC. Prediction of protein cellular attributes using pseudo-amino-acidcomposition. Proteins Struct Funct Genet. 2001;43:246-55.

41. Wang YC, Wang XB, Yang ZX, Deng NY. Prediction of enzyme subfamily class via pseudo amino acid composition by incorporating the conjoint triad feature. Protein Pept Lett. 2010;17:1441-9.

42. Chou KC. Using amphiphilic pseudo amino acid composition to predict enzyme subfamily classes. Bioinformatics. 2005;21:10-9.

43. Chou KC. Pseudo amino acid composition and its applications in bioinformatics, proteomics and system biology. Curr Proteomics. 2009:6:262-74.

44. Dubchak I, Muchnik I, Holbrook SR, Kim SH. Prediction of protein folding class using global description of amino acid sequence. Proc Natl Acad Sci U S A. 1995;92(19):8700-4.

45. Cai CZ, Han LY, Ji ZL, Chen X, Chen YZ. SVM-Prot: web-based support vector machine software for functional classification of a protein from its primary sequence. Nucl Acids Res. 2003:31(13):3692-7.

46. Govindan G, Nair AS. Composition, Transition and Distribution (CTD) - A dynamic feature for predictions based on hierarchical structure of cellular sorting. Hyderabad: India Conference (INDICON); 2011.

47. Loftin C, Ward SK. Spatial autocorrelation models for Galton's problem. Behav Sci Res. 1981;16:105-41.

48. Kawashima S, Kanehisa M. AAindex: amino acid index database. Nucl Acids Res. 2000;28:374.

49. Vapnik V. The nature of statistical learning theory. New York: Springer-Verlag Press; 2000.

50. Chen W, Feng PM, Lin H, Chou KC. IRSpot-PseDNC: identify recombination spots with pseudo dinucleotide composition. Nucl Acids Res. 2013;41:e68.

51. Lin H, Ding H. Predicting ion channels and their types by the di-peptide mode of pseudo amino acid composition. J Theor Biol. 2011:269:64-9.

52. Chou KC, Cai YD. Using functional domain composition and support vector machines for prediction of protein subcellular location. J Biol Chem. 2002: 277:45765-9.

53. Cai YD, Zhou GO, Chou KC. Support vector machines for predicting membrane protein types by using functional domain composition. Biophys J. 2003:84:3257-63.

54. Chen W, Lin H. Prediction of midbody, centrosome, and kinetochore proteins based on gene ontology information. Biochem Biophys Res Commun. 2010;401:382-4. 
55. Hayat M, Khan A. Predicting membrane protein types by fusing composite protein sequence features into pseudo amino acid composition. J Theor Biol. 2011;271:10-7.

56. Xiao X, Wang P, Chou KC. INR-PhysChem: a sequence-based predictor for identifying nuclear receptors and their subfamilies via physical-chemical property matrix. PLoS One. 2012;7:e30869.

57. Brown MPS, Grundy WN, Lin D, Cristianini N, Sugnet CW, Furey TS, Jr MA, Haussler D. Knowledge-based analysis of microarray gene expression data by using support vector machines. Proc Natl Acad Sci. 2000;97:262-7.

58. Ding $\mathrm{CHQ}$, Dubchak I. Multi-class protein fold recognition using support vector machines and neural networks. Bioinformatics. 2001;17:349-58.

59. Cristianini N, Shawe-Taylor J. An introduction to support vector machines and other kernel-based learning methods. UK: Cambridge University Press; 2000

60. Henderson J, Salzberg S, Fasman KH. Finding genes in DNA with a hidden markov model. J Comput Biol. 1996;4:127-41.

61. Baten A, Halgamuge SK, Chang B, Li J. Splice site identification using probabilistic parameters and SVM classification. BMC Bioinform. 2006;7:1-15.

62. Bradley AP. The use of the area under the ROC curve in the evaluation of machine learning algorithms. Pattern Recogn. 1997;30:1145-59.

63. Altschul SF, Gish W, Miller W, Myers E, Lipman D. Basic local alignment search tool. J Mol Biol. 1990:215(3):403-10.

64. Altschul SF, Madden TL, Schaffer AA, Zhang J, Zhang Z, Miller W, Lipman DJ. Gapped BLAST and PSI-BLAST: a new generation of protein database search programs. Nucl Acids Res. 1997;25(17):3389-402.

65. Boratyn GM, Schäffer AA, Agarwala R, Altschul SF, Lipman DJ, Madden TL. Domain enhanced lookup time accelerated BLAST. Biol Direct. 2012;7:12.

66. Zuo Y, Peng X, Wang K, Lin F, Li Y, Chen M. Expression patterns, mutation detection and RNA interference of Rhopalosiphum padi voltage-gated sodium channel genes. Sci Rep. 2016;6:30166.

67. Wondji CS, Dabire RK, Tukur Z, Irving H, Djouaka R, Morgan JC. Identification and distribution of a GABA receptor mutation conferring dieldrin resistance in the malaria vector Anopheles funestus in Africa. Insect Biochem Molec Biol. 2011;41:484-91.

68. Li F, Han Z. Mutations in acetylcholinesterase associated with insecticide resistance in the cotton aphid, Aphis gossypii Glover. Insect Biochem Molec Biol. 2004;34:397-405.

69. Silva AX, Jander G, Samaniego H, Ramsey JS, Figueroa CC. Insecticide resistance mechanisms in the green peach aphid Myzus persicae (Hemiptera: Aphididae) I: a transcriptomic survey. Plos One. 2012;7:e36366.

70. Belinato TA, Martins AJ. Insecticide Resistance and Fitness Cost. 2016. http:// dx.doi.org/10.5772/61826. pp 243-261.

71. Sparks TC, Nauen R. IRAC: mode of action classification and insecticide resistance management. Pestic Biochem Phys. 2015;121:122-8.

72. Prince DC, Drurey C, Zipfel C, Hogenhout SA. The leucine-rich repeat receptor-like kinase BRASSINOSTEROID INSENSITIVE1-ASSOCIATED KINASE1 and the cytochrome P450 PHYTOALEXIN DEFICIENT3 contribute to innate immunity to aphids in Arabidopsis. Plant Physiol. 2014;164(4):2207-19.

73. Mamidala P, Wijeratne AJ, Wijeratne S, Kornacker K, Sudhamalla B, RiveraVega L, Hoelmer A, Meulia T, Jones SC, Mittapalli O. RNA-Seg and molecular docking reveal multi-level pesticide resistance in the bed bug. BMC Genomics. 2012;13:6

74. Hassani O, Mansuelle P, Cestèle S, Bourdeaux M, Rochat H, Sampieri F. Role of lysine and tryptophan residues in the biological activity of toxin VII (Ts gamma) from the scorpion Tityus serrulatus. Eur J Biochem. 1999;260(1):76-86.

\section{Submit your next manuscript to BioMed Central and we will help you at every step:}

- We accept pre-submission inquiries

- Our selector tool helps you to find the most relevant journal

- We provide round the clock customer support

- Convenient online submission

- Thorough peer review

- Inclusion in PubMed and all major indexing services

- Maximum visibility for your research

Submit your manuscript at www.biomedcentral.com/submit 